\title{
Brain imaging biomarkers of carotid artery disease
}

\author{
Hediyeh Baradaran ${ }^{1}$, Ajay Gupta ${ }^{2,3}$ \\ ${ }^{1}$ Department of Radiology, University of Utah, Salt Lake City, UT, USA; ${ }^{2}$ Department of Radiology, ${ }^{3}$ Feil Family Brain and Mind Research Institute, \\ Weill Cornell Medicine, New York, NY, USA \\ Contributions: (I) Conception and design: All authors; (II) Administrative support: H Baradaran; (III) Provision of study materials or patients: H \\ Baradaran; (IV) Collection and assembly of data: H Baradaran; (V) Data analysis and interpretation: All authors; (VI) Manuscript writing: All authors; \\ (VII) Final approval of manuscript: All authors. \\ Correspondence to: Hediyeh Baradaran, MD. 30 N 1900 E \#1A141, Salt Lake City, UT 84135, USA. Email: hediyeh.baradaran@hsc.utah.edu.
}

\begin{abstract}
Extracranial carotid artery atherosclerotic disease is a major contributor to ischemic stroke. Carotid atherosclerotic disease can present with a spectrum of findings ranging from mild carotid intimamedia thickness to high-risk vulnerable carotid plaque features and carotid stenosis. Before leading to clinically overt stroke or transient ischemic attack, there may be other markers of downstream ischemia secondary to carotid atherosclerotic disease. In this review article, we will review some of the imaging findings that may be seen downstream to carotid artery disease on various imaging modalities, including hemodynamic and perfusional abnormalities which may be seen on CT, MR, or using other advanced imaging techniques, white matter hyperintensities on brain imaging, silent or covert brain infarctions, cerebral microbleeds, and regional and generalized cerebral volume loss. Many of these imaging findings are seen routinely on brain magnetic resonance imaging in patients without overt clinical symptoms. Despite frequently being asymptomatic, many of these imaging findings are also strongly associated with increased risk of future stroke, cognitive impairment, and even mortality. We will review the existing evidence underpinning the associations between these frequently encountered imaging findings and carotid artery atherosclerotic disease. Future validation of these imaging findings could lead to them being powerful biomarkers of cerebrovascular health.
\end{abstract}

Keywords: Carotid artery diseases; cerebrovascular disorders; cognitive dysfunction; magnetic resonance imaging; stroke

Submitted Feb 26, 2020. Accepted for publication May 10, 2020.

doi: 10.21037/atm-20-1939

View this article at: http://dx.doi.org/10.21037/atm-20-1939

\section{Introduction}

Carotid artery atherosclerosis is an established risk factor for cerebrovascular disease and stroke $(1,2)$. In addition to directly causing ischemic stroke, there are many additional deleterious downstream effects of carotid artery atherosclerosis that may be more covert but, nonetheless, are major contributors to patient morbidity and mortality. While these "silent" markers of carotid artery disease may not produce overt or easily identifiable clinical consequences, such as a large ischemic stroke, many are apparent on routine neurological imaging, often in asymptomatic patients. These findings can be seen on a variety of imaging examinations ranging from routine head CT or brain MR to advanced imaging techniques, including CT or MR perfusion or nuclear medicine techniques. Although some of these findings may be seen in otherwise asymptomatic patients, their presence may indicate cerebrovascular compromise, increased risk for future or recurrent stroke, or increased risk for vascular cognitive impairment (3-8). The downstream impact of extracranial carotid disease may be secondary to a wide variety of carotid disease phenotypes, ranging from mild increases in carotid intima media thickness to stenotic carotid plaques with high-risk features (9-13). While the degree of stenosis and features of the carotid plaque itself are critical factors to 
Table 1 Summary of imaging biomarkers of carotid atherosclerosis

\begin{tabular}{|c|c|c|c|}
\hline Imaging biomarker & Definition & Imaging technique & Associated risk \\
\hline $\begin{array}{l}\text { White matter } \\
\text { hyperintensities (WMH) }\end{array}$ & $\begin{array}{l}\text { T2/FLAIR hyperintensities in the } \\
\text { periventricular and subcortical } \\
\text { white matter }\end{array}$ & $\begin{array}{l}\text { Measured both quantitatively and } \\
\text { qualitatively, most commonly on } \\
\text { MR but can be seen on CT }\end{array}$ & $\begin{array}{l}\text { WMH associated with increased risk of } \\
\text { stroke, cognitive decline, dementia, and } \\
\text { death }\end{array}$ \\
\hline Cerebral microbleeds & $\begin{array}{l}\text { Small hemosiderin deposits } \\
\text { from microvascular leakage }\end{array}$ & $\begin{array}{l}\mathrm{T}^{*} \text {-weighted gradient echo or } \\
\text { susceptibility images on MR }\end{array}$ & $\begin{array}{l}\text { Associated with increased risk of } \\
\text { ischemic infarctions, intracerebral } \\
\text { hemorrhage, cognitive impairment, and } \\
\text { dementia }\end{array}$ \\
\hline
\end{tabular}

\#, this can use vasodilatory stimulus such as acetazolamide.

consider when making treatment decisions in patients with carotid artery disease, other imaging findings downstream from the carotid artery disease may also indicate additional "silent" effects of carotid artery disease (14-16). We will review brain imaging findings on both routine and advanced imaging that are associated with carotid artery atherosclerosis.

\section{Mechanisms}

Extracranial atherosclerotic disease accounts for $15-20 \%$ of all ischemic strokes (17). There are two main pathways for which strokes and transient ischemic attacks occur in patients with carotid artery disease (18). First, a stenosing plaque can limit downstream cerebral blood flow, resulting in hemodynamic alterations, hypoperfusion and potentially hypoxia and infarction. It is for this reason high-grade carotid stenosis is a well-established risk factor for stroke and why carotid endarterectomies are generally recommended for symptomatic patients with high-grade stenosis (19). In addition, the degree of carotid stenosis has long been included as the major determinant of treatment decisions for patients with carotid artery disease, at least in part, because it has served as the dominant inclusion criteria in multiple clinical trials $(16,20)$.

Alternatively, the carotid plaque itself may lead to an artery-to-artery thromboembolic event, resulting in infarction. High-risk plaque features including intraplaque hemorrhage, lipid rich necrotic core, plaque ulceration and other surface irregularities can lead to emboli extending from the plaque surface to distal cerebral arteries or arterioles $(15,21)$. The microembolic phenomena resulting from high risk plaque features has become an important consideration in evaluating patients at high-risk for stroke. These high-risk plaque features can be characterized with both routine and advanced imaging techniques.

It is likely that a significant proportion of ischemic strokes secondary to carotid disease arise from a synergy between hemodynamic and embolic risk factors, since impaired perfusion from flow limitations from a stenosing plaque may make an embolic event that may have been transient, into a cerebral infarction (18). These mechanisms which individually and together account for a large number of ischemic strokes also contribute to structural and microstructural changes to the brain which are evident on imaging but may be difficult to detect clinically (Table 1).

\section{Markers of hemodynamic and perfusional abnormalities}

One of the most direct methods by which carotid artery disease may cause downstream effects is through the hypoperfusion secondary to stenotic or turbulent flow from flow-limiting plaque (18). Many hemodynamic risk factors 
are associated with stroke in carotid artery disease including impairment of cerebrovascular reserve and increased oxygen extraction fraction $(7,8)$. Imaging measurements of hemodynamic alterations downstream from carotid artery disease have been studied as a potential biomarker for increased risk of cerebrovascular disease, even in asymptomatic patients $(22,23)$. Severe, flow-limiting carotid stenosis results in a reduction in cerebral perfusion pressure. In response to a reduced perfusion pressure, cerebral blood flow may be maintained by (I) vasodilation of the cerebral vasculature and/or (II) increase in oxygen extraction (24). When faced with constant reductions in perfusion pressure, vessel autoregulation will lead to maximal dilatation to maintain cerebral blood flow so with any further reduction in cerebral blood flow, there is an increased risk for stroke.

Cerebrovascular reserve is a measure of the ability of vessels to vasodilate when faced with reduced cerebral perfusion pressure (25). There are two main methods for measuring cerebrovascular reserve (7). First, imaging may attempt to directly measure cerebral blood flow to the brain with flow-sensitive imaging techniques including positron emission tomography, other nuclear medicine techniques, CT or MR perfusion both before and after a vasodilatory stimulus $(26,27)$. This approach directly measures CBF at a tissue level. Alternatively, transcranial Doppler can measure flow velocities distal to an area of stenosis or occlusion both before and after a vasodilatory stimulus $(23,28,29)$. Vasodilatory stimuli may be increasing $\mathrm{CO}_{2}$ levels with breath-holding or inhalation of $\mathrm{CO}_{2}$ gases (30) or with pharmacologic methods, such as acetazolamide $(22,31)$.

CT perfusion (CTP) is a well-studied and straightforward method for assessing cerebrovascular reserve. Studies have shown that asymptomatic patients with carotid artery stenosis who have abnormal cerebrovascular reserve on CTP are more likely to develop ipsilateral ischemic events (32). In addition, studies have found that hemodynamic changes on CTP can be reversed with carotid artery stenting in both symptomatic and asymptomatic patients (Figure 1) (33-35). Though CTP is widely available, the risks associated with radiation exposure and the need for iodinated contrast limit its ability to be widely used as a screening examination. There are also a number of MR based examinations which can be used to evaluate cerebrovascular hemodynamics and perfusion in the setting of carotid artery stenosis. MR perfusion can be measured with dynamic susceptibility contrast perfusion imaging which uses a $\mathrm{T} 2 *$-weighted echo planar sequence after the administration of intravenous contrast to measure various perfusion parameters. Findings are variable, but many believe that high-grade stenosis may result in elevated MTT (36). Another popular method for measuring MR perfusion is arterial spin labeling (ASL) which can measure perfusion non-invasively, without intravenous contrast by using magnetically-labeled water protons in flowing blood as a tracer. ASL can characterize CBF in patients with ICA occlusion (37) and identify deficits in cerebrovascular reserve in patients with ICA stenosis (38) and occlusion (39).

A comprehensive systematic review and meta-analysis of 13 studies using transcranial Doppler or nuclear medicine techniques to study cerebrovascular reserve with almost 1,000 patients found that there was a significant positive relationship between impaired cerebrovascular reserve and development of future stroke [odds ratio (OR) 3.86; 95\% confidence interval (CI), 1.99-7.48] (7). Importantly, this statistically significant relationship persisted with asymptomatic patients, meaning that even completely asymptomatic patients with carotid artery disease who demonstrated impairment in their cerebrovascular reserve were at a higher risk of developing a future stroke.

Positron emission tomography (PET) based measures of cerebral hemodyn amics including measuring oxygen extraction fraction (OEF) using ${ }^{15} \mathrm{O}$ can also be used to measure hemodynamic alterations in the setting of carotid artery stenosis. In the setting of severe carotid stenosis and a reduction in cerebral blood flow, there may be a compensatory increase in oxygen extraction (so called "misery perfusion"). This is thought to occur mostly in endstages of hemodynamic failure so is usually more pronounced in already symptomatic patients. Indeed, in patients with highgrade carotid artery stenosis $(\geq 70 \%)$, a systematic review and meta-analysis found a significant positive relationship between abnormal oxygen extraction and future ipsilateral stroke (OR 6.04; 95\% CI, 2.58-14.12) (8). A study of asymptomatic patients did not find a significant association between increased OEF and future stroke (40). While measuring OEF may not be the most effective method for assessing asymptomatic patients with carotid artery stenosis, it can be a tool to identify those who are experiencing end-stage hemodynamic alterations and may be at higher risk for future cerebrovascular ischemia. It also further highlights the downstream hemodynamic alterations experienced in patients with steno-occlusive carotid artery disease. Although using $\mathrm{O}^{15}$ methods can be difficult to implement in clinical practice, significant efforts are underway to develop MRI-based techniques to measure the cerebral metabolic rate of oxygen $\left(\mathrm{CMRO}^{2}\right)$ and $\mathrm{OEF}$ as imaging 


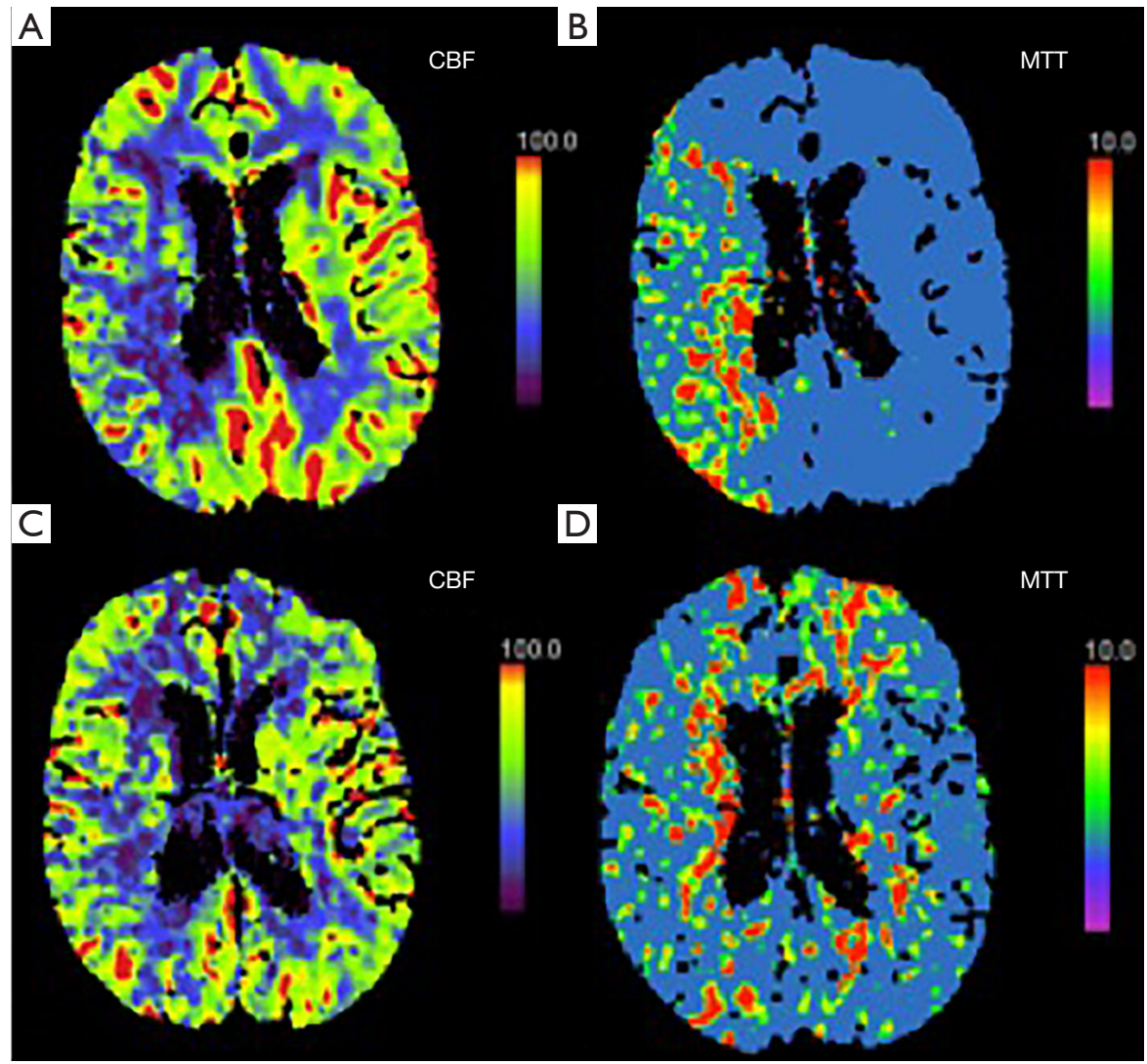

Figure 1 An 87-year-old male with near complete occlusion of his right internal carotid artery (not shown) with evidence of decreased cerebral blood flow (CBF) and increased mean transit time (MTT) throughout the right cerebral hemisphere on pre-operative CT Perfusion $(\mathrm{A}, \mathrm{B})$ indicating decreased perfusion. After carotid artery stenting, both the CBF and MTT are more symmetric with the contralateral hemisphere $(\mathrm{C}, \mathrm{D})$.

stroke risk markers (41-43).

Some of the methods for measuring cerebrovascular reserve or oxygen extraction fraction require advanced imaging techniques or the use of vasodilatory stimuli that are not always routinely performed. Despite this, many studies have demonstrated that carotid artery stenosis results in downstream hemodynamic alterations, even in asymptomatic patients, and these hemodynamic alterations may increase the risk for future or recurrent stroke.

\section{White matter hyperintensities (WMH)}

Leukoaraiosis, or WMH, are frequently encountered "incidentally" on routine brain MR imaging with a prevalence ranging from $11-21 \%$ to $94 \%$ depending on age $(44,45)$. These brain lesions are most commonly seen as T2 and T2/FLAIR (fluid attenuating inversion recovery) hyperintensities in the periventricular and subcortical white matter. WMH can be measured both quantitatively with various mapping tools and qualitatively using visual rating scales on routine brain MR imaging. Initially thought to be mostly incidental, the presence of white matter hyperintensities has been shown to increase risk of stroke, cognitive decline, dementia, and death $(3,4,6)$. The increased risk of stroke, dementia, and cognitive decline is present in the general population, not just high-risk populations. Multiple mechanistic explanations for the association of $\mathrm{WMH}$ and cognitive decline and dementia exist, including the possibility that $\mathrm{WMH}$ are a reflection of the association of vascular risk factors or exist concomitantly with ischemic damage leading to dementia. Both increasing age and hypertension are associated with the presence of $\mathrm{WMH}$, but carotid artery atherosclerosis may also play a role in the development of WMH. 
The exact association between carotid atherosclerosis and $\mathrm{WMH}$ is uncertain with multiple studies demonstrating mixed results. There are a number of studies that demonstrate an association of white matter hyperintensities in the setting of carotid atherosclerosis, even independent of age and hypertension $(46,47)$. Some studies show an association with carotid atherosclerosis and deeper, periventricular WMH, but not with subcortical WMH (48). Increased thickness of carotid intima-media thickness, a surrogate marker for atherosclerosis measured on ultrasound, has also been shown to be associated with increased WMH in multiple asymptomatic cohorts (13). There may be stronger associations with WMH and increased CIMT, than with carotid stenosis. There are inconsistent and mixed results demonstrating hemispheric differences in WMH directly downstream from carotid artery stenosis which makes a direct causative association difficult to establish (49).

There is evidence of increased white matter hyperintensities ipsilateral to "unstable" or high-risk carotid plaque $(50,51)$. The association of increased white matter hyperintensities downstream to unstable carotid plaque were observed regardless of degree of carotid stenosis. These findings may indicate that the thromboembolic plaque activity contributes more heavily to the presence of white matter hyperintensities, rather than hypoperfusion effects. This may be supported by other studies which have shown that the degree of WMH are not associated with $\mathrm{CBF}$ in patients with severe carotid stenosis, indicating that WMH may not be seen as a direct result of hypoperfusion (52).

Carotid artery stiffness is an important marker of arterial health and is generally measured on carotid US. Many studies have demonstrated an association between carotid artery stiffness and increased burden of WMH. The presence of carotid artery stiffness on ultrasound has been shown to accurately predict WMH 20 years later (53). Additional studies have shown that larger carotid artery diameters or other findings of extracranial carotid artery remodeling are also associated with the burden of WMH (54).

There is evidence that there is damage to neuronal integrity downstream to carotid artery disease, even before white matter hyperintensities are evident. In asymptomatic patients, there is evidence of microstructural damage and decreased neuronal integrity downstream from carotid artery disease (55). This is evident on sensitive diffusion tensor imaging studies which demonstrate lower fractional anisotropy and higher mean diffusivity values ipsilateral to carotid artery atherosclerosis. These changes in fractional anisotropy and mean diffusivity are indicative of microstructural neuronal damage and have been shown to be associated with cognitive dysfunction and can even be detected in normal-appearing white matter (56), suggesting that neuronal injury may occur before any visible findings on standard MR imaging. Additional studies have shown that larger plaque calcification volume is also associated with worse microstructural integrity measured on diffusion tensor imaging (57).

WMH are frequently noted on routine brain MR imaging and are strongly associated with increased risk of stroke, cognitive decline, and even death. The development of WMH is likely multifactorial with both age and hypertension being established risk factors. There is some evidence, however, that carotid artery disease may also play a role in the development of $\mathrm{WMH}$, given that carotid artery atherosclerosis as measured by CIMT and carotid plaque features and are associated with increased WMH. This association seems to be unrelated to the degree of carotid stenosis, suggesting a negligible role for hypoperfusion in the development of WMH.

\section{Silent brain infarctions}

Distinct from WMH, silent brain infarctions (SBIs) are also visualized "incidentally" on routine brain MR imaging. They are defined as infarctions identified with MR imaging which did not present as clinically overt symptoms or signs and were not previously diagnosed as a transient ischemic attack or stroke. While these infarctions, by definition, did not have clinically obvious stroke-like symptoms, they may still be associated with more subtle deficits, both physically and cognitively. For this reason, many are steering away from the calling them "silent" as they can result in functional or cognitive changes, with the term "covert" brain infarcts now increasingly being used (58). Similar to WMH, SBIs are commonly detected on routine MR imaging and are seen in about $20 \%$ of healthy elderly people and up to $50 \%$ of patients with more risk factors (59). SBIs are important to recognize and report on because they more than double the risk of subsequent stroke and dementia $(6,60,61)$, increase perioperative and long-term stroke risk and mortality in patients undergoing carotid endarterectomy (62), and are strongly associated with cognitive impairment $(5,63,64)$.

Unfortunately, there is no universal definition of imaging criteria for SBI. There are two main categories for covert brain infarctions: (I) lacunar cavitary infarctions, which 

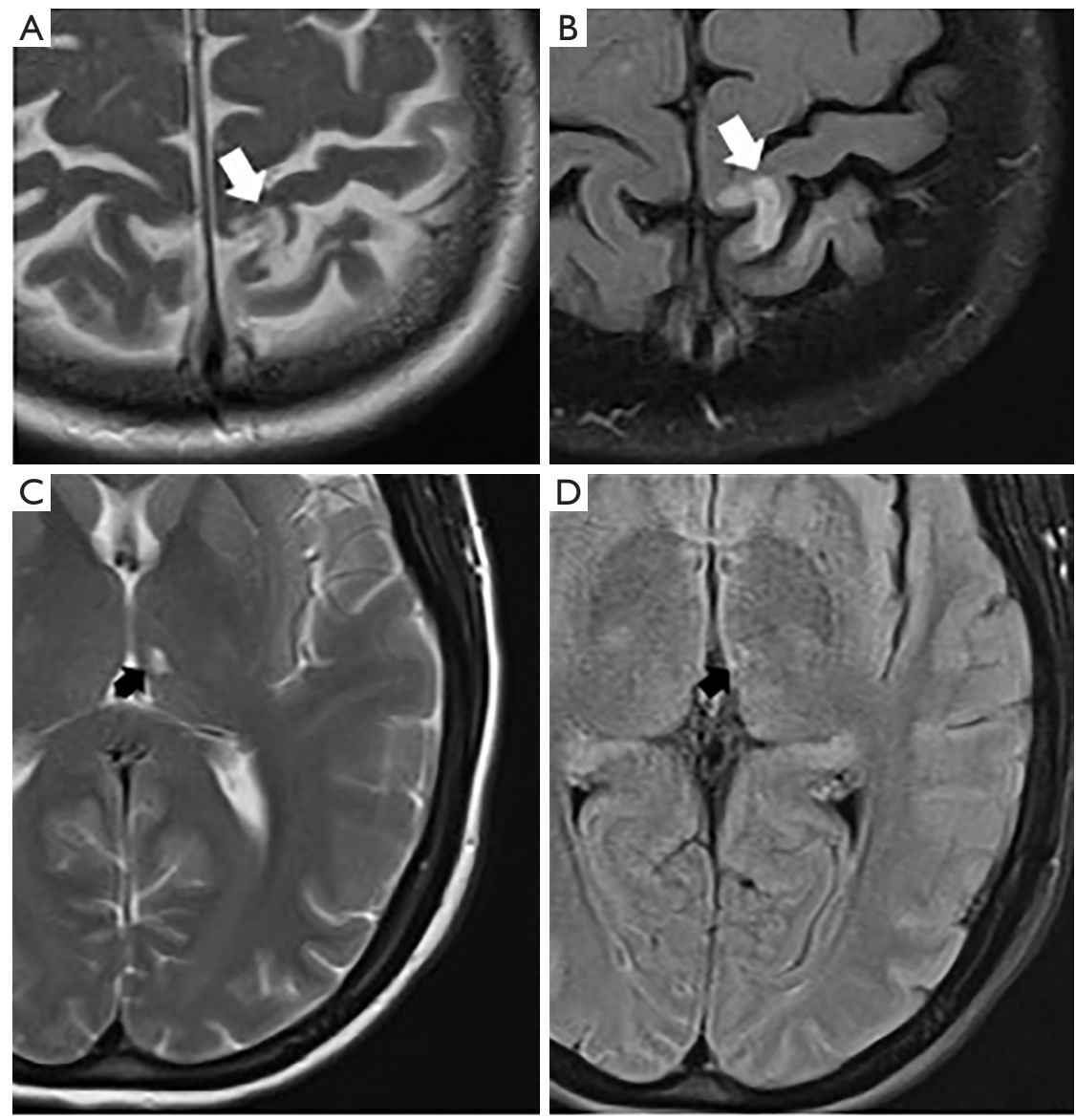

Figure 2 Silent brain infarctions. (A,B) A 78-year-old asymptomatic male with left carotid artery stenosis (not pictured) and encephalomalacia and gliosis in the left parietal lobe (white arrow) consistent with cortical infarction; (C,D) a 63-year-old asymptomatic female with chronic lacunar infarction in the left thalamus.

are most strongly associated with hypertensive small vessel disease and (II) cortical infarctions which may have the same pathophysiology as overt infarctions (Figure 2). While there is some variability in the definition of the SBIs, the lacunar subtype of SBIs is most common and are generally defined as a focus in the deep gray or white matter which follows CSF signal on all pulse sequences with a gliotic T2FLAIR hyperintense rim $(65,66)$. These can be confused with dilated Virchow-Robin spaces which are similarly of CSF signal intensity on all pulse sequences, but do not have a T2-FLAIR hyperintense rim. The cortical subtype of SBIs can occur in any non-eloquent regions of the cerebral cortex. Furthermore, SBIs can also be seen in the cerebellum and brainstem.

There are a number of studies demonstrating the strong association between carotid artery atherosclerosis and the presence of SBIs, in both symptomatic and asymptomatic cohorts $(47,61,67-71)$. A study of asymptomatic patients with carotid artery atherosclerosis found a higher prevalence of SBIs downstream from ICA disease compared to the contralateral side (72). There was a significant difference in cortical SBIs between hemispheres. The lacunar subtype, however, did not show a significant difference between sides. Other preliminary data similarly demonstrated those with asymptomatic carotid stenosis had a greater proportion of cortical SBIs (73). Additionally, there is evidence of an association of SBIs with high-risk plaque features, likely secondary to increased likelihood of emboli $(61,74,75)$. Similar to $\mathrm{WMH}$, increasing carotid lumen diameter and increased carotid stiffness is also found to be associated with increasing prevalence of lacunar infarcts (70).

SBIs likely arise from a variety of potentially independent mechanisms. One major potential cause of SBIs is small vessel vasculopathy, which is supported by the fact that 

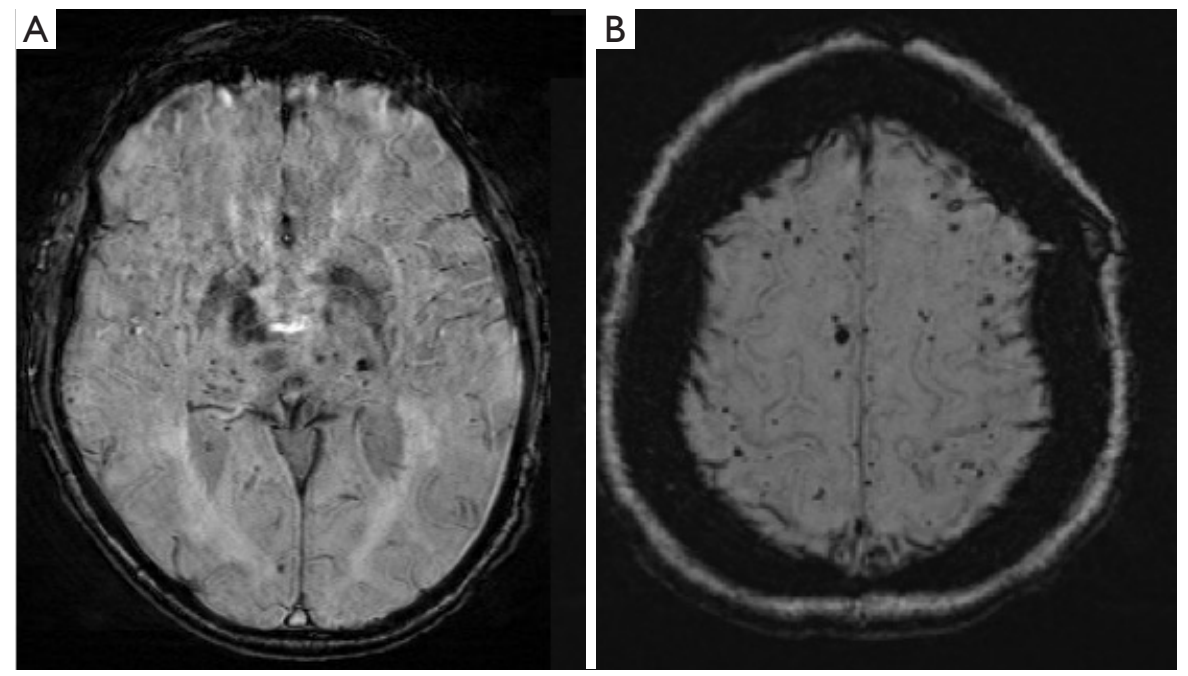

Figure 3 Cerebral microbleeds. (A) A 74-year-old male with multiple foci of susceptibility hypointensity predominantly in the bilateral thalami; (B) a 69-year-old male with multiple foci of susceptibility hyperintensity in a peripheral, lobar pattern, more commonly associated with cerebral amyloid angiopathy.

most SBIs occur in deep gray and white matter where there are smaller vessels and that SBIs are closely associated with leukoaraiosis. There is also evidence that SBIs can be caused by embolic phenomenon and hypoperfusion, both of which can be seen in the setting of carotid artery disease (76-79). Since the association between carotid artery disease and stroke is very well-established, the association between carotid artery disease and certain types of SBIs would also necessarily be strong, given that many of these infarctions have a similar pathophysiology.

\section{Cerebral microbleeds (CMBs)}

$\mathrm{CMBs}$ are another frequently encountered incidental finding on routine brain imaging. They are small hypointense foci on $\mathrm{T} 2{ }^{*}$-weighted gradient echo and susceptibility images on MR. On histopathology, these foci represent small hemosiderin deposits from microvascular leakage. Similar to other previously discussed incidental findings on $\mathrm{MR}, \mathrm{CMBs}$ are associated with increased risk of ischemic infarctions, intracerebral hemorrhage, cognitive impairment, and dementia (80-84). CMBs are generally asymptomatic and indicate the presence of hemorrhage-prone cerebral small vessel disease. They are most commonly seen in older individuals with an increasing prevalence as patients age, from around $6.5 \%$ at age 45 to 50 to $35.7 \%$ at age 80 (85). They most commonly are thought to be secondary to hypertension and cerebral amyloid angiopathy. CMBs in the deep or infratentorial regions are generally associated with hypertensive angiopathy while lobar and more peripheral CMBs are more closely associated with underlying cerebral amyloid angiopathy (Figure 3). Although hypertension, advancing age, and amyloid angiopathy are well-established risk factors for CMBs, other processes may also be associated with the development of CMBs, including carotid atherosclerosis.

Many studies have identified a link between carotid atherosclerosis and the presence of CMBs. In almost all studies, carotid atherosclerosis was more strongly associated with deep and infratentorial CMBs, as opposed to lobar CMBs which are more commonly associated with cerebral amyloid angiopathy. There have been several studies indicating an association between carotid atherosclerosis, indicated by carotid intima-media thickness and the presence of deep CMBs $(86,87)$. The presence of carotid stenosis $\geq 25 \%$ is associated with the presence of both overall CMBs and at deep and mixed locations (12). Additional studies have shown an association between the presence of carotid artery plaque and CMBs. For example, calcification in the ICA was found to be an independent risk factor for deep CMBs (88). In addition, some studies have shown that there is an association between higher-risk fatty carotid plaques and CMBs $(89,90)$. Furthermore, multiple studies have shown that increased carotid artery stiffness, which is also associated with hypertension, may contribute to the development of CMBs, especially in the deep location (91). 

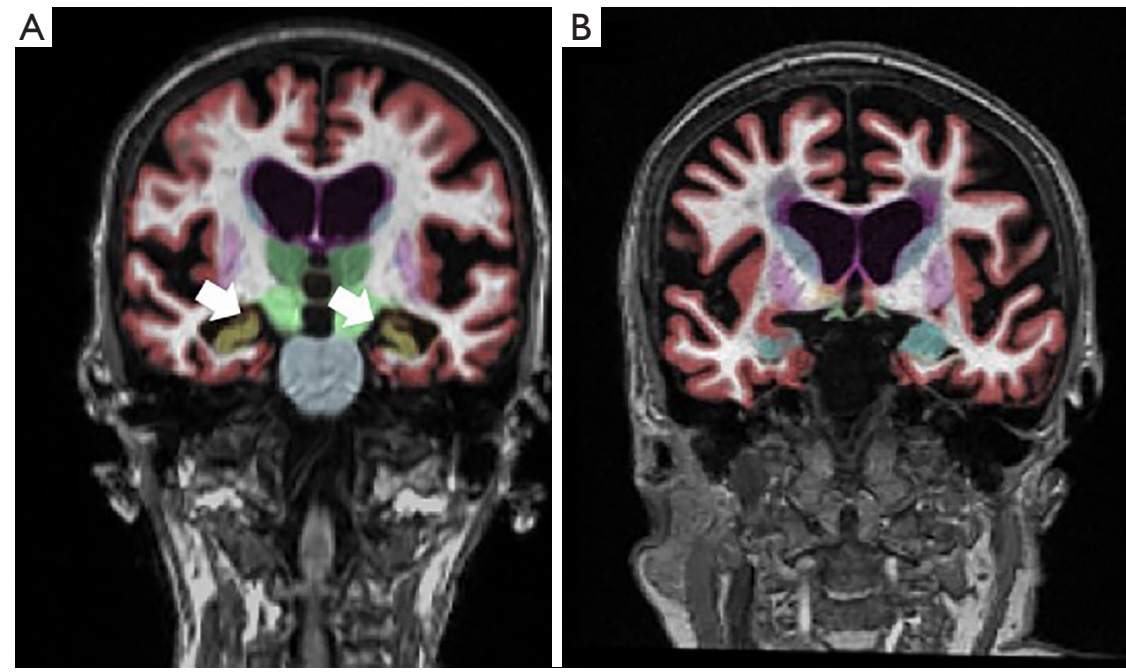

Figure 4 Cerebral volume loss. (A) A 73-year-old female with predominant hippocampal volume loss (arrows); (B) a 77-year-old female with diffuse cerebral volume loss.

These studies suggest CMBs are likely multifactorial and can be present in asymptomatic patients with carotid artery disease.

\section{Brain volume}

Decreased cerebral volume and atrophy is also frequently visualized on routine MR imaging (Figure 4). This finding is nonspecific and is associated with normal aging (92), dementia including Alzheimer's disease (93), cerebrovascular disease including stroke (94), cardiovascular disease including myocardial infarction (95), and other neurodegenerative disorders (96). The presence of brain atrophy is strongly associated with cognitive impairment (97). Carotid atherosclerosis has also been associated with decreased cerebral volume and brain atrophy, with support from multiple cross-sectional studies. The mechanism underpinning the association between carotid atherosclerosis and decreased cerebral volumes has not been definitely established, though arterial stiffness from carotid atherosclerosis is thought to lead to relative cerebral hypoperfusion and then brain atrophy $(98,99)$. Many studies have shown that both increased CIMT and carotid stenosis are associated with decreased relative total brain and cortical gray matter volume $(13,100,101)$. Increased CIMT is also associated with increased progression of total brain and gray matter volume (102). Furthermore, severe or bilateral carotid stenosis has also been shown to be related to progression of global, cortical, and subcortical atrophy (100). Additional studies suggest the degree of carotid plaque burden may also be associated with cerebral atrophy (103). Furthermore, higher risk carotid plaque characteristics, including ulceration or surface irregularity or heterogeneous echotexture on ultrasound, were also associated with brain atrophy, even after adjustment for age and other cerebrovascular risk factors (67). In addition, larger volume of overall plaque calcification is also associated with decreased brain volume (57).

In addition to overall decreased brain volume and decreased cortical gray matter volume, some studies have also shown an association of carotid atherosclerosis to decreased hippocampal volumes (104). Lower hippocampal volumes are also independently associated with cognitive impairment and dementia.

\section{Conclusions}

In addition to the ischemic stroke, carotid artery disease is associated with many downstream markers of cerebrovascular ischemia that can be evaluated on both routine and advanced imaging. Most of these imaging findings are not clinically obvious and can be considered "silent", though there is increasing evidence of their longterm negative impact on patient health outcomes. These imaging findings may be powerful tools in risk stratifying patients with carotid artery atherosclerosis. Using MR or CT perfusion or other nuclear medicine studies to evaluate cerebrovascular reserve or oxygen extraction fraction may 
be a helpful tool to identify those who may be more likely to benefit from carotid endarterectomy. The presence of other findings on brain MR, including white matter hyperintensities, silent brain infarctions, and cerebral microbleeds, may influence medical management of risk factors for stroke. Future research efforts are warranted to standardize definitions and validate the use of these neuroimaging findings as potentially powerful biomarkers of brain health in patients with carotid disease.

\section{Acknowledgments}

Funding: AG is in part supported by National Institutes of Health grants (R01HL144541 and R21HL145427).

\section{Footnote}

Provenance and Peer Review: This article was commissioned by the Guest Editor (Dr. Kosmas I. Paraskevas) for the series "Carotid Artery Stenosis and Stroke: Prevention and Treatment Part I" published in Annals of Translational Medicine. The article was sent for external peer review organized by the Guest Editor and the editorial office.

Conflicts of Interest: Both authors have completed the ICMJE uniform disclosure form (available at http://dx.doi. org/10.21037/atm-20-1939). The series "Carotid Artery Stenosis and Stroke: Prevention and Treatment Part I" was commissioned by the editorial office without any funding or sponsorship. HB reports grants from Association of University Radiologists-General Electric Radiology Research Fellowship, during the conduct of the study. AG reports non-financial support from GE Healthcare and non-financial support from Siemens Medical Solutions USA, Inc., outside the submitted work. The authors have no other conflicts of interest to declare.

Ethical Statement: The authors are accountable for all aspects of the work in ensuring that questions related to the accuracy or integrity of any part of the work are appropriately investigated and resolved.

Open Access Statement: This is an Open Access article distributed in accordance with the Creative Commons Attribution-NonCommercial-NoDerivs 4.0 International License (CC BY-NC-ND 4.0), which permits the noncommercial replication and distribution of the article with the strict proviso that no changes or edits are made and the original work is properly cited (including links to both the formal publication through the relevant DOI and the license). See: https://creativecommons.org/licenses/by-nc-nd/4.0/.

\section{References}

1. de Weerd M, Greving JP, de Jong AW, et al. Prevalence of asymptomatic carotid artery stenosis according to age and sex: systematic review and metaregression analysis. Stroke 2009;40:1105-13.

2. Brott TG, Halperin JL, Abbara S, et al. 2011 ASA/ACCF/ AHA/AANN/AANS/ACR/ASNR/CNS/SAIP/SCAI/ SIR/SNIS/SVM/SVS guideline on the management of patients with extracranial carotid and vertebral artery disease: A report of the American college of cardiology foundation/American heart association task force on practice guidelines, and the American stroke association, American association of neuroscience nurses, American association of neurological surgeons, American college of radiology, American society of neuroradiology, congress of neurological surgeons, society of atherosclerosis imaging and prevention, society for cardiovascular angiography and interventions, society of interventional radiology, society of neurointerventional surgery, society for vascular medicine, and society for vascular surgery developed in collaboration with the American academy of neurology and society of cardiovascular computed tomography. J Am Coll Cardiol 2011;57:e16-e94.

3. Debette S, Markus HS. The clinical importance of white matter hyperintensities on brain magnetic resonance imaging: systematic review and meta-analysis. BMJ 2010;341.

4. Debette S, Beiser A, DeCarli C, et al. Association of MRI markers of vascular brain injury with incident stroke, mild cognitive impairment, dementia, and mortality. Stroke 2010;41:600-6.

5. Vermeer SE, Prins ND, den Heijer T, et al. Silent brain infarcts and the risk of dementia and cognitive decline. $\mathrm{N}$ Engl J Med 2003;348:1215-22.

6. Vermeer SE, Hollander M, van Dijk EJ, et al. Silent brain infarcts and white matter lesions increase stroke risk in the general population. Stroke 2003;34:1126-9.

7. Gupta A, Chazen JL, Hartman M, et al. Cerebrovascular reserve and stroke risk in patients with carotid stenosis or occlusion: a systematic review and meta-analysis. Stroke 2012;43:2884-91.

8. Gupta A, Baradaran H, Schweitzer A, et al. Oxygen extraction fraction and stroke risk in patients with carotid 
stenosis or occlusion: a systematic review and metaanalysis. AJNR Am J Neuroradiol 2014;35:250-5.

9. Wendell CR, Zonderman AB, Metter EJ, et al. Carotid Intimal Medial Thickness Predicts Cognitive Decline Among Adults Without Clinical Vascular Disease. Stroke 2009;40:3180.

10. Spence JD. Asymptomatic carotid stenosis. Circulation 2013;127:739-42.

11. Saam T, Hetterich H, Hoffmann V, et al. Meta-analysis and systematic review of the predictive value of carotid plaque hemorrhage on cerebrovascular events by magnetic resonance imaging. J Am Coll Cardiol 2013;62:1081-91.

12. Romero JR, Preis SR, Beiser A, et al. Carotid atherosclerosis and cerebral microbleeds: the Framingham Heart Study. J Am Heart Assoc. 2016;5:e002377.

13. Romero JR, Beiser A, Seshadri S, et al. Carotid Artery Atherosclerosis, MRI Indices of Brain Ischemia, Aging, and Cognitive Impairment. Stroke 2009;40:1590.

14. Gupta A, Kesavabhotla K, Baradaran H, et al. Plaque echolucency and stroke risk in asymptomatic carotid stenosis: a systematic review and meta-analysis. Stroke 2015;46:91-7.

15. Gupta A, Baradaran H, Schweitzer AD, et al. Carotid Plaque MRI and Stroke Risk. Stroke 2013;44:3071-7.

16. Collaborators* NASCET. Beneficial effect of carotid endarterectomy in symptomatic patients with high-grade carotid stenosis. N Engl J Med 1991;325:445-53.

17. Raman G, Moorthy D, Hadar N, et al. Management strategies for asymptomatic carotid stenosis: a systematic review and meta-analysis. Ann Intern Med 2013;158:676-85.

18. Caplan LR, Hennerici M. Impaired clearance of emboli (washout) is an important link between hypoperfusion, embolism, and ischemic stroke. Arch Neurol 1998;55:1475-82.

19. Pearson TA, Blair SN, Daniels SR, et al. AHA guidelines for primary prevention of cardiovascular disease and stroke: 2002 update: consensus panel guide to comprehensive risk reduction for adult patients without coronary or other atherosclerotic vascular diseases. Circulation 2002;106:388-91.

20. Mantese VA, Timaran CH, Chiu D, et al. The carotid revascularization endarterectomy versus stenting trial (CREST) stenting versus carotid endarterectomy for carotid disease. Stroke 2010;41:S31-S4.

21. Baradaran H, Al-Dasuqi K, Knight-Greenfield A, et al. Association between Carotid Plaque Features on CTA and Cerebrovascular Ischemia: A Systematic Review and Meta-
Analysis. AJNR Am J Neuroradiol 2017;38:2321-6.

22. Silvestrini M, Vernieri F, Pasqualetti $P$, et al. Impaired cerebral vasoreactivity and risk of stroke in patients with asymptomatic carotid artery stenosis. JAMA 2000;283:2122-7.

23. King A, Serena J, Bornstein NM, et al. Does impaired cerebrovascular reactivity predict stroke risk in asymptomatic carotid stenosis? A prospective substudy of the asymptomatic carotid emboli study. Stroke 2011;42:1550-5.

24. Kimiagar I, Bass A, Rabey J, et al. Long-term follow-up of patients with asymptomatic occlusion of the internal carotid artery with good and impaired cerebral vasomotor reactivity. Eur J Neurol 2010;17:1285-90.

25. Markus H, Cullinane M. Severely impaired cerebrovascular reactivity predicts stroke and TIA risk in patients with carotid artery stenosis and occlusion. Brain 2001;124:457-67.

26. Ogasawara K, Ogawa A, Terasaki K, et al. Use of cerebrovascular reactivity in patients with symptomatic major cerebral artery occlusion to predict 5-year outcome: comparison of xenon-133 and iodine-123-IMP singlephoton emission computed tomography. J Cereb Blood Flow Metab 2002;22:1142-8.

27. Isozaki M, Arai Y, Kudo T, et al. Clinical implication and prognosis of normal baseline cerebral blood flow with impaired vascular reserve in patients with major cerebral artery occlusive disease. Ann Nucl Med 2010;24:371-7.

28. Kleiser B, Widder B. Course of carotid artery occlusions with impaired cerebrovascular reactivity. Stroke 1992;23:171-4.

29. Reinhard M, Gerds TA, Grabiak D, et al. Cerebral dysautoregulation and the risk of ischemic events in occlusive carotid artery disease. J Neurol 2008;25 5:1182.

30. Vernieri F, Pasqualetti P, Passarelli F, et al. Outcome of carotid artery occlusion is predicted by cerebrovascular reactivity. Stroke 1999;30:593-8.

31. Kuroda S, Houkin K, Kamiyama H, et al. Long-term prognosis of medically treated patients with internal carotid or middle cerebral artery occlusion: can acetazolamide test predict it? Stroke 2001;32:2110-6.

32. Liu S, Cai J, Ge F, et al. The risk of ischemic events increased in patients with asymptomatic carotid stenosis with decreased cerebrovascular reserve. J Investig Med 2017;65:1028-32.

33. Merckel LG, Van der Heijden J, Jongen LM, et al. Effect of Stenting on Cerebral CT Perfusion in Symptomatic and Asymptomatic Patients with Carotid Artery Stenosis. 
AJNR Am J Neuroradiol 2012;33:280-5.

34. Trojanowska A, Drop A, Jargiello T, et al. Changes in cerebral hemodynamics after carotid stenting: evaluation with CT perfusion studies. J Neuroradiol 2006;33:169-74.

35. Waaijer A, van Leeuwen MS, van Osch MJ, et al. Changes in cerebral perfusion after revascularization of symptomatic carotid artery stenosis: CT measurement. Radiology 2007;245:541-8.

36. Bozzao A, Floris R, Gaudiello F, et al. Hemodynamic modifications in patients with symptomatic unilateral stenosis of the internal carotid artery: evaluation with MR imaging perfusion sequences. AJNR Am J Neuroradiol 2002;23:1342-5.

37. Bokkers RP, Bremmer JP, Van Berckel BN, et al. Arterial spin labeling perfusion MRI at multiple delay times: a correlative study with $\mathrm{H} 215 \mathrm{O}$ positron emission tomography in patients with symptomatic carotid artery occlusion. J Cereb Blood Flow Metab 2010;30:222-9.

38. Bokkers RP, van Osch MJ, van der Worp HB, et al. Symptomatic carotid artery stenosis: impairment of cerebral autoregulation measured at the brain tissue level with arterial spin-labeling MR imaging. Radiology 2010;256:201-8.

39. Bokkers RP, van Osch MJ, Klijn CJ, et al. Cerebrovascular reactivity within perfusion territories in patients with an internal carotid artery occlusion. J Neurol Neurosurg Psychiatry 2011;82:1011-6.

40. Powers WJ, Derdeyn C, Fritsch S, et al. Benign prognosis of never-symptomatic carotid occlusion. Neurology 2000;54:878-82.

41. Zhang J, Cho J, Zhou D, et al. Quantitative susceptibility mapping-based cerebral metabolic rate of oxygen mapping with minimum local variance. Magn Reson Med 2018;79:172-9.

42. Cho J, Zhang S, Kee Y, et al. Cluster analysis of time evolution (CAT) for quantitative susceptibility mapping (QSM) and quantitative blood oxygen level-dependent magnitude (qBOLD)-based oxygen extraction fraction $(\mathrm{OEF})$ and cerebral metabolic rate of oxygen (CMRO2) mapping. Magn Reson Med 2020;83:844-57.

43. Donahue MJ, Achten E, Cogswell PM, et al. Consensus statement on current and emerging methods for the diagnosis and evaluation of cerebrovascular disease. J Cereb Blood Flow Metab 2018;38:1391-417.

44. Ylikoski A, Erkinjuntti T, Raininko R, et al. White matter hyperintensities on MRI in the neurologically nondiseased elderly: analysis of cohorts of consecutive subjects aged 55 to 85 years living at home. Stroke 1995;26:1171-7.
45. Garde E, Mortensen EL, Krabbe K, et al. Relation between age-related decline in intelligence and cerebral white-matter hyperintensities in healthy octogenarians: a longitudinal study. Lancet 2000;356:628-34.

46. Pico F, Dufouil C, Lévy C, et al. Longitudinal study of carotid atherosclerosis and white matter hyperintensities: the EVA-MRI cohort. Cerebrovasc Dis 2002;14:109-15.

47. Moroni F, Ammirati E, Magnoni M, et al. Carotid atherosclerosis, silent ischemic brain damage and brain atrophy: a systematic review and meta-analysis. Int J Cardiol 2016;223:681-7.

48. de Leeuw FE, de Groot JC, Bots ML, et al. Carotid atherosclerosis and cerebral white matter lesions in a population based magnetic resonance imaging study. J Neurol 2000;247:291-6.

49. Baradaran H, Mtui E, Richardson J, et al. Hemispheric differences in leukoaraiosis in patients with carotid artery stenosis: a systematic review. Clin Neuroradiol 2017;27:7-13.

50. Altaf N, Morgan PS, Moody A, et al. Brain white matter hyperintensities are associated with carotid intraplaque hemorrhage. Radiology 2008;248:202-9.

51. Altaf N, Daniels L, Morgan P, et al. Cerebral white matter hyperintense lesions are associated with unstable carotid plaques. Eur J Vasc Endovasc Surg 2006;31:8-13.

52. Patankar T, Widjaja E, Chant H, et al. Relationship of deep white matter hyperintensities and cerebral blood flow in severe carotid artery stenosis. Eur J Neurol 2006;13:10-6.

53. de Havenon A, Wong KH, Elkhetali A, et al. Carotid Artery Stiffness Accurately Predicts White Matter Hyperintensity Volume 20 Years Later: A Secondary Analysis of the Atherosclerosis Risk in the Community Study. AJNR Am J Neuroradiol 2019;40:1369-73.

54. Rundek T, Della-Morte D, Gardener H, et al. Relationship between carotid arterial properties and cerebral white matter hyperintensities. Neurology 2017;88:2036-42.

55. Baradaran H, Mtui EE, Richardson JE, et al. White Matter Diffusion Abnormalities in Carotid Artery Disease: A Systematic Review and Meta-Analysis. J Neuroimaging 2016;26:481-8.

56. Maillard P, Seshadri S, Beiser A, et al. Effects of systolic blood pressure on white-matter integrity in young adults in the Framingham Heart Study: A cross-sectional study. Lancet Neurol 2012;11:1039-47.

57. Bos D, Vernooij MW, Elias-Smale SE, et al. Atherosclerotic calcification relates to cognitive function and to brain changes on magnetic resonance imaging. 
Alzheimers Dement 2012;8:S104-S11.

58. Chabriat H. Covert Brain Infarcts: Look at Extra and Intracranial Vessels. Stroke 2020;51:2-3.

59. Vermeer SE, Koudstaal PJ, Oudkerk M, et al. Prevalence and risk factors of silent brain infarcts in the populationbased Rotterdam Scan Study. Stroke 2002;33:21-5.

60. Gupta A, Giambrone AE, Gialdini G, et al. Silent brain infarction and risk of future stroke: a systematic review and meta-analysis. Stroke 2016;47:719-25.

61. Jayasooriya G, Thapar A, Shalhoub J, et al. Silent cerebral events in asymptomatic carotid stenosis. J Vasc Surg 2011;54:227-36.

62. Pini R, Faggioli G, Longhi M, et al. The detrimental impact of silent cerebral infarcts on asymptomatic carotid endarterectomy outcome. J Vasc Surg 2016;64:15-24.

63. Longstreth W, Bernick C, Manolio TA, et al. Lacunar infarcts defined by magnetic resonance imaging of 3660 elderly people: the Cardiovascular Health Study. Arch Neurol 1998;55:1217-25.

64. Matsui T, Arai H, Yuzuriha T, et al. Elevated plasma homocysteine levels and risk of silent brain infarction in elderly people. Stroke 2001;32:1116-9.

65. Zhu Y-C, Dufouil C, Tzourio C, et al. Silent brain infarcts: a review of MRI diagnostic criteria. Stroke 2011;42:1140-5.

66. Fanning JP, Wong AA, Fraser JF. The epidemiology of silent brain infarction: a systematic review of populationbased cohorts. BMC Med 2014;12:119.

67. Manolio TA, Burke GL, O'Leary DH, et al. Relationships of Cerebral MRI Findings to Ultrasonographic Carotid Atherosclerosis in Older Adults. Arterioscler Thromb Vasc Biol 1999;19:356.

68. Inoue K, Matsumoto M, Shono T, et al. Increased intima media thickness and atherosclerotic plaques in the carotid artery as risk factors for silent brain infarcts. J Stroke Cerebrovasc Dis 2007;16:14-20.

69. Das RR, Seshadri S, Beiser AS, et al. Prevalence and correlates of silent cerebral infarcts in the Framingham offspring study. Stroke 2008;39:2929-35.

70. Brisset M, Boutouyrie P, Pico F, et al. Large-vessel correlates of cerebral small-vessel disease. Neurology 2013;80:662-9.

71. Kakkos SK, Sabetai M, Tegos T, et al. Silent embolic infarcts on computed tomography brain scans and risk of ipsilateral hemispheric events in patients with asymptomatic internal carotid artery stenosis. J Vasc Surg 2009;49:902-9.

72. Baradaran H, Gialdini G, Mtui E, et al. Silent brain infarction in patients with asymptomatic carotid artery atherosclerotic disease. Stroke 2016;47:1368-70.

73. Wright C, Dong C, Yoshita M, et al. Abstract 3166: Asymptomatic Internal Carotid Artery Stenosis is Associated with More Cortical Subclinical Brain Infarcts: the Northern Manhattan Study (NOMAS). Stroke 2012;43:A3166.

74. Geroulakos G, Domjan J, Nicolaides A, et al. Ultrasonic carotid artery plaque structure and the risk of cerebral infarction on computed tomography. J Vasc Surg 1994;20:263-6.

75. El-Barghouti N, Nicolaides A, Tegos T, et al. The relative effect of carotid plaque heterogeneity and echogenicity on ipsilateral cerebral infarction and symptoms of cerebrovascular disease. Int Angiol 1996;15:300-6.

76. Horowitz DR, Tuhrim S, Weinberger JM, et al. Mechanisms in lacunar infarction. Stroke 1992;23:325-7.

77. Bogousslavsky J, Regli F, Maeder P. Intracranial largeartery disease and lacunar infarction. Cerebrovasc Dis 1991;1:154-9.

78. Zhu C, Norris J, editors. Lacunar infarction and carotid stenosis. Boston, MA: Annals of Neurology, 1991.

79. Miao Q, Paloneva T, Tuisku S, et al. Arterioles of the lenticular nucleus in CADASIL. Stroke 2006;37:2242-7.

80. Poels MM, Ikram MA, van der Lugt A, et al. Cerebral microbleeds are associated with worse cognitive function: the Rotterdam Scan Study. Neurology 2012;78:326-33.

81. Qiu C, Cotch M, Sigurdsson S, et al. Cerebral microbleeds, retinopathy, and dementia: the AGESReykjavik Study. Neurology 2010;75:2221-8.

82. Vernooij M, Heeringa J, de Jong GJ, et al. Cerebral microbleed preceding symptomatic intracerebral hemorrhage in a stroke-free person. Neurology 2009;72:763-5.

83. Charidimou A, Kakar P, Fox Z, et al. Cerebral microbleeds and recurrent stroke risk: systematic review and metaanalysis of prospective ischemic stroke and transient ischemic attack cohorts. Stroke 2013;44:995-1001.

84. Cordonnier C, Van der Flier W, Sluimer J, et al. Prevalence and severity of microbleeds in a memory clinic setting. Neurology 2006;66:1356-60.

85. Poels MM, Vernooij MW, Ikram MA, et al. Prevalence and risk factors of cerebral microbleeds: an update of the Rotterdam scan study. Stroke 2010;41:S103-S6.

86. Chung CP, Chou KH, Chen WT, et al. Location of Cerebral Microbleeds And Their Association with Carotid Intima-media Thickness: A Community-based Study. Sci Rep 2017;7:12058.

87. Zhao FF, Gao HY, Gao Y, et al. A Correlational Study 
on Cerebral Microbleeds and Carotid Atherosclerosis in Patients with Ischemic Stroke. J Stroke Cerebrovasc Dis 2018;27:2228-34.

88. Chung PW, Park KY, Kim JM, et al. Carotid artery calcification is associated with deep cerebral microbleeds. Eur Neurol 2014;72:60-3.

89. Saba L, Sanfilippo R, di Martino M, et al. Volumetric analysis of carotid plaque components and cerebral microbleeds: a correlative study. J Stroke Cerebrovasc Dis 2017;26:552-8.

90. Saba L, Montisci R, Raz E, et al. Association between carotid artery plaque type and cerebral microbleeds. AJNR Am J Neuroradiol 2012;33:2144-50.

91. Ding J, Mitchell GF, Bots ML, et al. Carotid arterial stiffness and risk of incident cerebral microbleeds in older people: the Age, Gene/Environment Susceptibility (AGES)-Reykjavik study. Arterioscler Thromb Vasc Biol 2015;35:1889-95.

92. Kennedy KM, Erickson KI, Rodrigue KM, et al. Agerelated differences in regional brain volumes: a comparison of optimized voxel-based morphometry to manual volumetry. Neurobiol Aging 2009;30:1657-76.

93. McDonald C, McEvoy L, Gharapetian L, et al. Regional rates of neocortical atrophy from normal aging to early Alzheimer disease. Neurology 2009;73:457-65.

94. Grau-Olivares M, Bartrés-Faz D, Arboix A, et al. Mild cognitive impairment after lacunar infarction: voxelbased morphometry and neuropsychological assessment. Cerebrovasc Dis 2007;23:353-61.

95. Grubb NR, Fox KA, Smith K, et al. Memory impairment in out-of-hospital cardiac arrest survivors is associated with global reduction in brain volume, not focal hippocampal injury. Stroke 2000;31:1509-14.

Cite this article as: Baradaran H, Gupta A. Brain imaging biomarkers of carotid artery disease. Ann Transl Med 2020;8(19):1277. doi: 10.21037/atm-20-1939
96. Cardenas VA, Studholme C, Gazdzinski S, et al. Deformation-based morphometry of brain changes in alcohol dependence and abstinence. Neuroimage 2007;34:879-87.

97. Chao L, Mueller S, Buckley S, et al. Evidence of neurodegeneration in brains of older adults who do not yet fulfill MCI criteria. Neurobiology of aging 2010;31:368-77.

98. Scuteri A, Brancati AM, Gianni W, et al. Arterial stiffness is an independent risk factor for cognitive impairment in the elderly: a pilot study. J Hypertens 2005;23:1211-6.

99. Nation DA, Sweeney MD, Montagne A, et al. Bloodbrain barrier breakdown is an early biomarker of human cognitive dysfunction. Nat Med2019;25:270-6.

100.Muller M, van der Graaf Y, Algra A, et al. Carotid atherosclerosis and progression of brain atrophy: the SMART-MR study. Ann Neurol 2011;70:237-44.

101. Cardenas VA, Reed B, Chao LL, et al. Associations among vascular risk factors, carotid atherosclerosis, and cortical volume and thickness in older adults. Stroke 2012;43:2865-70.

102. Sabayan B, van Buchem MA, Sigurdsson S, et al. Cardiac and Carotid Markers Link With Accelerated Brain Atrophy: The AGES-Reykjavik Study (Age, Gene/ Environment Susceptibility-Reykjavik). Arterioscler Thromb Vasc Biol 2016;36:2246-51.

103. Kin T, Yamano S, Sakurai R, et al. Carotid atherosclerosis is associated with brain atrophy in Japanese elders. Gerontology 2007;53:1-6.

104. Baradaran H, Demissie S, Himali JJ, et al. The progression of carotid atherosclerosis and imaging markers of dementia. Alzheimers Dement (N Y) 2020;6:e12015. 